\section{Commentary}

\section{ROY MEADOW}

Department of Paediatrics and Child Health, St James's University Hospital, Leeds

Nephrologists will be intrigued by this case history, and as the pathological findings conflict with current beliefs about Henoch-Schönlein nephritis some will ask whether this child really had Henoch-Schönlein syndrome. The clinical features of the illness, which followed an upper respiratory tract infection and which involved the alimentary system, the joints, the kidneys and the skin, seem typical but the pathognomic feature of Henoch-Schönlein syndrome is the rash, for it is the characteristics of the rash that allow the clinical diagnosis. It is unfortunate that no photograph of the rash is available, but understandable since the authors had no particular reason to take a photograph at the time when they believed this to be a straightforward case of HenochSchönlein syndrome. We have to accept the accuracy of their description and the validity of these experienced clinicians saying that it was characteristic of Henoch-Schönlein purpura.

Thirty years ago $\beta$ haemolytic streptococcal infections were a common precipitant of HenochSchönlein purpura; they are a less common cause now as the incidence of streptococcal infection in the industrialised countries has decreased. This child had such an infection and an appropriate antistreptolysin 0 titre response. But, the ensuing illness was not a classic post-streptococcal acute glomerulonephritis: serum C3 complement values remained normal and the general features of the ensuing illness, particularly the rash, were characteristic of Henoch-Schönlein syndrome. The twist in this tale is that although the nephritis that accompanied the Henoch-Schönlein syndrome was clinically appropriate, the renal biopsy did not show the one feature that has come to be regarded as pathonognomic of Henoch-Schönlein nephritis. For though on light microscopy there may be a wide variety of different histological appearances, most of them variations of a diffuse mesangial glomerulonephritis, the pathonognomic feature of Henoch-Schönlein nephritis is the immunofluorescent finding of $\operatorname{IgA}$ deposits in the mesangium. The constancy of these deposits together with the observation that some children with Henoch-Schönlein syndrome have raised serum $\operatorname{IgA}$, and an increased proportion of $\operatorname{IgA}$ bearing lymphocytes, as well as the finding of IgA immune complexes has led to a widespread acceptance that IgA immune complexes are a cause of the nephritis. The similarity of the immunofluorescent findings and the immunological observations to those of Berger disease (mesangial IgA nephropathy) which often presents with haematuria, has led to the suggestion that Henoch-Schönlein nephritis and Berger disease are the same and that people with Berger disease could be considered as having HenochSchönlein syndrome without the rash.

Now this report seems to suggest that HenochSchönlein syndrome and a nephritis may occur in someone with specific deficit of $\operatorname{IgA}$ and that the features, including the nephritis, may occur in the absence of IgA deposits. Therefore, some will wonder whether the $\operatorname{IgA}$ abnormalities of HenochSchönlein syndrome are merely epiphenomena and irrelevant to the aetiology; others will question whether this girl did have Henoch-Schönlein syndrome-and many of us will wish that we had had the opportunity to examine the child and see the rash ourselves when it first occurred.

\title{
Symptomatic vitamin E deficiency in cystic fibrosis
}

\section{A M E BYE, D P R MULLER, J WILSON, V M WRIGHT, AND M B MEARNS}

Queen Elizabeth Hospital for Children and Institute of Child Health, University of London, London

SUMMARY A girl with cystic fibrosis who developed a neurological syndrome probably secondary to vitamin $\mathrm{E}$ deficiency at the age of 10 years is described. The severity of the deficiency and the early development of neurological features probably result from reduced intraluminal bile salt concentrations in addition to the pancreatic insufficiency.
Reduced serum concentrations of vitamin $\mathrm{E}$ have been documented in cystic fibrosis, ${ }^{1}$ and there is now some evidence to indicate that vitamin $E$ is necessary for normal neurological function. ${ }^{2}$ Neuropathological changes ${ }^{3}$ and a spinocerebellar syndrome have been described in adults with cystic fibrosis and vitamin $E$ deficiency. ${ }^{45}$

We report a girl with cystic fibrosis who had undetectable serum concentrations of vitamin $\mathrm{E}$ and 